\title{
Nanoscale Grain Boundary Dissociation: Role of Shockley Partial Dislocations
}

\author{
D.L. Medlin*, D. Cohen*, G. Lucadamo*, and S.M. Foiles** \\ * Sandia National Laboratories, MS 9161, Livermore, CA 94551 \\ **Sandia National Laboratories, MS 1411, Albuquerque, NM 87185
}

Many interfaces in low stacking fault energy (SFE) metals relax by emitting stacking faults that extend the structural perturbation of the interface over several planes normal to the interface [1-3]. In some cases, the arrangement of faults is sufficiently regular to form a distinct crystallographic phase. For instance, $\Sigma=3$ boundaries with facets near to $\{112\}$ reconstruct in low SFE metals to form a narrow $(\sim 1 \mathrm{~nm})$ layer of material with the rhombohedral 9R stacking arrangement [4,5]. The 9R stacking sequence, $\mathbf{a b c / b c a / c a b}$, can be related to FCC stacking by inserting an intrinsic stacking fault every third close-packed plane.

The 9R stacking arrangement is also found at $\{111\} /\{112\}$ facets at $90^{\circ}<110>$ boundaries in gold [6]. An HRTEM image showing one such boundary is illustrated in Figure 1. Here the positions of the faults are indicated by the white lines every three planes. Within the dissociated region the $\{111\}$ lattice fringes are locally bent through approximately $7^{\circ}$. A similar relaxation is found in atomistic simulations for the boundary (see Figure 2).

The presence of 9R stacking at these two distinct types of interface can be understood by considering the dislocation structure of the boundaries. The structures of $\langle 110\rangle$ tilt boundaries with misorientations between $50.5^{\circ}$ and $109.5^{\circ}$ can be modeled as arrays of pure-edge $\left(90^{\circ}\right)$ and mixed $\left(30^{\circ}\right)$ character Shockley partial dislocations, where the important parameter is the ratio of these two types of dislocation [6]. This approach provides insight into key geometrical features of the relaxations at both types of interface, including the distribution of stacking faults and the bending of planes near the boundaries. In the $\Sigma=3$ interface, the ratio of $90^{\circ}: 30^{\circ}$ dislocations is $1: 2$ [7], whereas in the $\{111\} /\{112\}$ interface the ratio is reversed, $2: 1$. In both cases, separation of the $90^{\circ}$ and $30^{\circ}$ dislocations produces the $9 \mathrm{R}$ sequence by introducing a stacking fault every third plane. This is illustrated schematically in Figure 3 for the $\{111\} /\{112\}$ interface. As we will discuss, this approach can be generalized to explain other polytypic stacking arrangements observed at dissociated boundaries of different orientation. These results demonstrate the important role of Shockley partial dislocations as fundamental elements of interfacial structure in FCC metals.

References:

[1] W. Krakow and D.A. Smith, Ultramicroscopy 22 (1987) 47.

[2] K.L. Merkle, Colloque de Physique 51 (1) (1990) C1:251.

[3] J.D. Rittner and D.N. Seidman, Physical Review B 54 (10) (1996) 6999.

[4] F. Ernst et al. Physical Review Letters 69 (4) (1992) 620.

[5] U. Wolf et al. Philosophical Magazine A 66 (1992) 295.

[6] D.L. Medlin et al., Acta materialia 49 (2001) 3689.

[7] C.B. Carter et al., Materials Science Forum 207-209 (1996) 209.

[8] This research is supported by the U.S. Department of Energy, in part by the Office of Basic Energy Sciences, under contract number DE-AC04-94-AL85000 


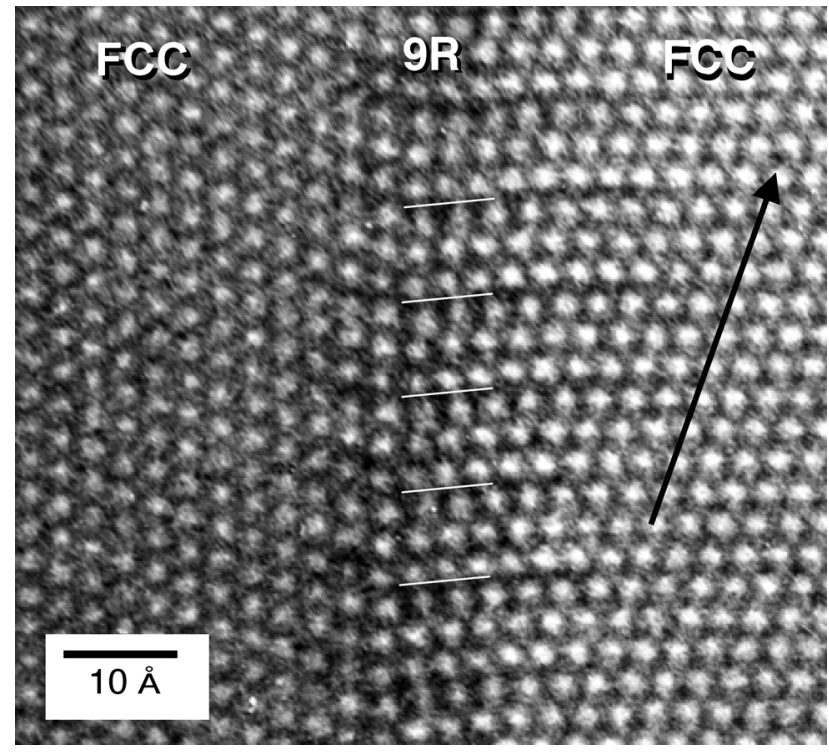

FIG 1. HRTEM micrograph of a $\{111\} /\{112\}$ interface in gold. The white lines, which are inclined at an angle of $7^{\circ}$, indicate the position and approximate extent of the faults.

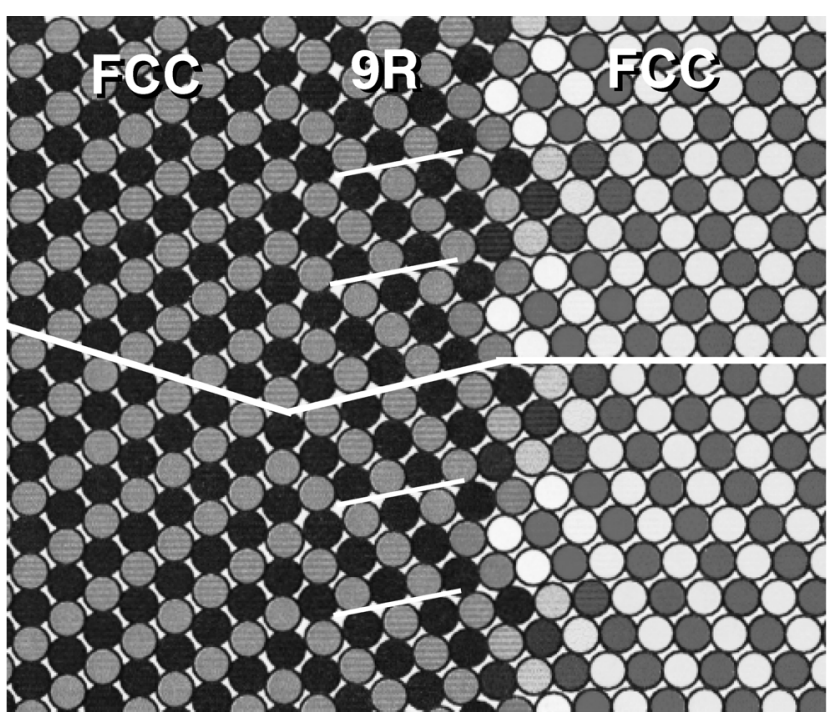

FIG. 2 Simulation of the $\{111\} /\{112\}$ interface calculated using an Embedded Atom Method (EAM) potential. The positions of stacking faults in the dissociated layer are indicated by the white lines.
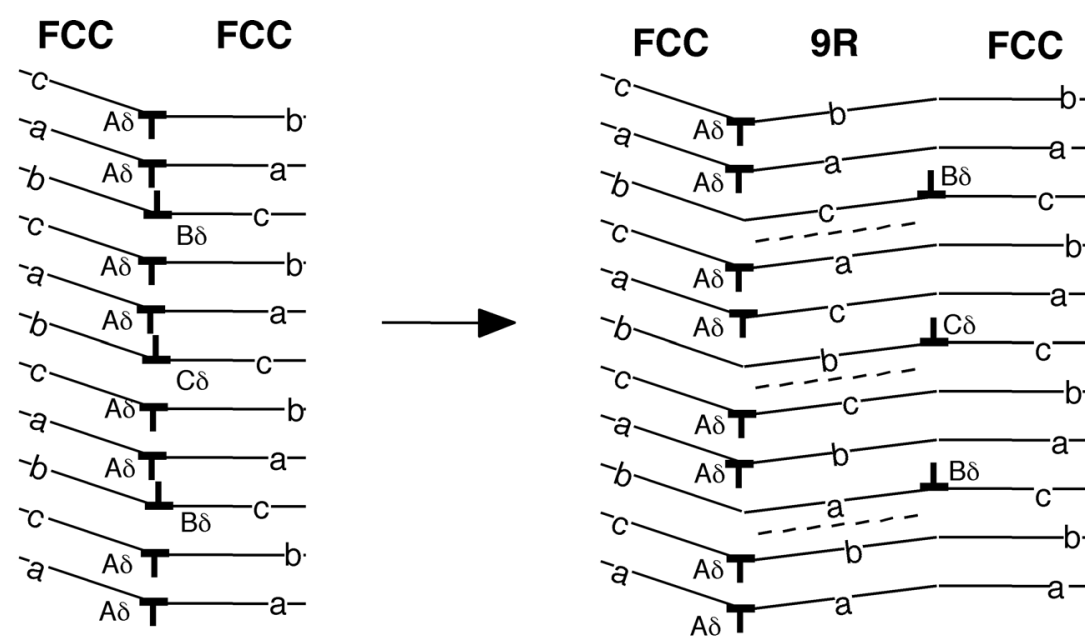

FIG. 3. Schematic illustrating how the 9R stacking is formed at the $\{111\} /\{112\}$ interface by the separation of the $30^{\circ}(\mathbf{B} \boldsymbol{\delta}$ and $\mathbf{C} \boldsymbol{\delta})$ and $90^{\circ}$ Shockleys (Aס) that comprise the interface. Here, the array of $30^{\circ}$ Shockleys is calculated to introduce a local plane bending of $6.7^{\circ}$. 